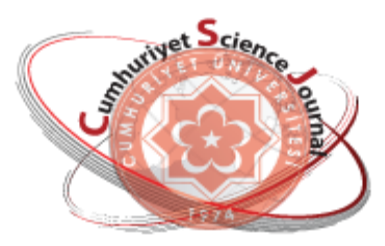

e-ISSN: $2587-246 X$

ISSN: $2587-2680$

\section{Cumburiyet Scionce Journal \\ esj}

Cumhuriyet Sci. J., Vol.40-2(2019) 299-304

\title{
Generalized Derivations of Hyperlattices
}

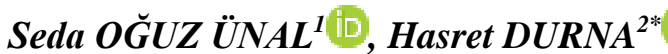 \\ ${ }^{1}$ Cumhuriyet University, Education Faculty, Department of Science and Education, Sivas, Turkey \\ ${ }^{2}$ Cumhuriyet University, Faculty of Science, Department of Mathematics, Sivas, Turkey
}

\begin{abstract}
In this paper the notion of generalized derivation for a hyperlattice is introduced and some basic properties of them are derived.

2010 Mathematics Subject Classification: 06B35; 06B75.

Keywords: Generalized derivations, Hyperlattice.
\end{abstract}

\section{Hiperlatisler Üzerinde Genelleştirilmiş Türevler}

Özet. $\mathrm{Bu}$ makalede hiperlatisler üzerinde genelleştirilmiş türev kavramı tanıtıldı ve bunların bazı temel özellikleri elde edildi.

Anahtar Kelimeler: Genelleştirilmiş türevler, Hiperlatis.

\section{INTRODUCTION AND PRELIMINARIES}

Firstly, Marty introduced the notion of hyperstructure in [1] at 8th Congress of Scandinavian Mathematicians. Normally, the composition of two elements is an element in classical algebraic structures, but the composition of two elements is a set in algebraic hyperstructures. After this study, many authors studied this subject. The many concepts in pure and applied mathematics were applied to hyperstructures [2,3]. There come out many kinds of hyperalgebras such as hypergroups in $[4,5]$, hyperrings in [6,7] etc. In [8], Konstantinidou and Mittas introduced hyperlattices and in [9] superlattices in [9] (for more details see [10] and [11]). In particular some interesting results of the theory of hyperlattices studied by Rasouli and Davvaz in $[12,13]$.

Derivations in rings and near-rings have been studied by many mathematicians in several ways [14, 15]. Bresar [16] introduced the generalized derivation in rings and many mathematicians studied on this concept. N. O. Alshehri applied the notion of generalized derivation in ring theory to lattices [17]. Now, we define the notion of derivation on hyperlattice. In this paper we aim to generalize some results given in [17] and [18] to generalized derivations of hyperlattices. In this way, we define generalized derivation on hyperlattice and give an example.

In this section, we first recall some definitions and basic results (for more detailed information see $[10,12,13])$. 
Definition 1.1 ([13]) Let $L$ be a nonempty set and $V: L \times L \rightarrow P^{*}(L)$ be a hyperoperation, where $P(L)$ is a power set of $L$ and $P^{*}(L)=P(L)-\emptyset$ and $\wedge: L \times L \rightarrow L$ be an operation. Then $(L, \vee, \wedge)$ is a hyperlattice if

(1) for all $a \in L, a \in a \vee a, a \wedge a=a$;

(2) for all $a, b \in L, a \vee b=b \vee a, a \wedge b=b \wedge a$;

(3) for all $a, b, c \in L,(a \vee b) \vee c=a \vee(b \vee c) ;(a \wedge b) \wedge c=a \wedge(b \wedge c)$;

(4) for all $a, b \in L, a \in[a \wedge(a \vee b)] \cap[a \vee(a \wedge b)]$;

(5) if $a \in a \vee b$ for all $a, b \in L_{\text {, then }} a \wedge b=b$.

Let $A$ and $B$ be nonempty subsets of $L$ then, $A \wedge B=\{a \wedge b \mid a \in A, b \in B\}$, $A \vee B=\{a \vee b \mid a \in A, b \in B\}$.

Let $L$ be a hyperlattice. For each $x, y \in L$, we define two relations on $L$ as follows: $(x, y) \in \leq$ if and only if $x=x \wedge y,(x, y) \in \leqslant$ if and only if $y \in x \vee y$. For all nonempty subsets $A$ and $B$ of $L$, we define $A \leq B$ if there exist $a \in A$ and $b \in B$ such that $a \leq b$.

A zero of a hyperlattice $L$ is an element 0 with $0 \leq x$ for all $x \in L$. A unit 1 , satisfies $x \leq 1$ for all $x \in L$, so it can be seen that there are at most one zero and at most one unit. A bounded hyperlattice is one that has both 0 and 1 . In a bounded hyperlattice $L, y$ is a complement of $x$ if $x \wedge y=0$ and $1 \in x \vee y$. The set of complement elements of $x$ is denoted by $x^{\circ}$. A complemented hyperlattice is a bounded hyperlattice in which every element has at least one complement.

Definition 1.2 ([3]) An element $a \in L$ is called a scalar element if the set a $\vee x$ for all $x \in L$ has only one element.

Proposition 1.3 ([13]) Let $(L, \vee, \wedge)$ be a hyperlattice. Then the following hold:

(1) $\leq=\leqslant$ and $(L, \leq)$ is a poset. Also we can replace Definition 1.1 (4) by $x \in x \wedge(x \vee y)$ for all $x, y \in L$;

(2) $x \wedge y \leq x, y \leq x \vee y$ for all $x, y \in L$;

(3) $X \subseteq(X \vee X) \cap(X \wedge X)$ for a nonempty subset $X_{\text {of }} L$;

(4) $X \vee(Y \vee Z)=(X \vee Y) \vee Z$ and $X \wedge(Y \wedge Z)=(X \wedge Y) \wedge Z$ for all nonempty subsets $X, Y, Z$ of $L$;

(5) If $x \leq y$, then $x \wedge z \leq y \wedge z$ for all $x, y, z \in L$;

(6) If $x, y \in x \vee y$, then $x=y$, so $x \vee y=L$ implies that $x=y$ for all $x, y \in L$;

(7) If $x \vee y=\{0\}$, then $x=y=0$ for all $x, y \in L$;

(8) If 0 is a scalar element of $L$, then $0 \vee 0=0, x \vee 0=\{x\}$ for all $x \in L$.

Definition 1.4 ([13]) A subhyperlattice of a hyperlattice $L$ is a nonempty subset of $L$ which is closed under the hyperoperation $\vee$ and operation $\wedge$ as defined in $L$.

Definition 1.5 ([13]) A hyperlattice $(L, \wedge, \vee, 0,1)$ is said to be a distributive if for every $a, b, c \in L, a \wedge(b \vee c)=(a \wedge b) \vee(a \wedge c)$ is hold. 
Definition 1.6 ([13]) Let $\left(L_{y} \wedge, \mathrm{V}\right)$ be a hyperlattice and I be a nonempty subset of $L$. Then I is called a hyperideal of $L$ when:

(i) I is a subhyperlattice;

(ii) $x \in I$ and $y \in L$ imply $x \wedge y \in I$.

Definition 1.7 Let $L$ be a hyperlattice. A mapping $d: L \rightarrow L$ such that, for all $x, y \in L$, we have

(1) $d(x \vee y) \subseteq d(x) \vee d(y),(2) d(x \wedge y) \in(d(x) \wedge y) \vee(x \wedge d(y))$

is said to be a derivation on $\mathrm{L}$, and the pair $(\mathrm{L}, \mathrm{d})$ is said to be a differential hyperlattice, or more precisely, a hyperlattice with a derivation. If the map $d$ such that $d(x \vee y)=d(x) \vee d(y)$ for all $x, y \in L$ and satisfies the condition (2), then $d$ is called a strong derivation of $L$. In this case, the pair ( $L, d)$ is called a strongly differential hyperlattice

\section{GENERALIZED DERIVATION OF HYPERLATTICES}

In this section we define generalized derivation and strong generalized derivation of hyperlattice and give examples. Through this section $L$ will denote a bounded hyperlattice and 0 be scalar element of $L$ unless otherwise specified.

Definition 2.1 A mapping $D: L \rightarrow L$ is called a generalized derivation on $L$ if there exists a derivation $d: L \rightarrow L$ such that

(1) $D(x \vee y) \subseteq D(x) \vee D(y)$

(2) $D(x \wedge y) \in(D(x) \wedge y) \vee(x \wedge d(y))$

for all $x, y \in L$. The pair $(L, D)$ is said to be a differential hyperlattice or is said to be hyperlattice with generalized derivation. The map $D$ is called strong generalized derivation if $D(x \vee y)=D(x) \vee D(y)$ and satisfies the condition (2). Then the pair $(L, D)$ is called a strongly differential hyperlattice.

Example 2.2. Let $L=\{0, a, b, 1\}$ and define $\Lambda$ and $v$ by the following Cayley tables

\begin{tabular}{|c|c|c|c|c|}
\hline$\wedge$ & 0 & $a$ & $b$ & 1 \\
\hline 0 & 0 & 0 & 0 & 0 \\
\hline$a$ & 0 & $a$ & 0 & $a$ \\
\hline$b$ & 0 & 0 & $b$ & $b$ \\
\hline 1 & 0 & $a$ & $b$ & 1 \\
\hline
\end{tabular}

\begin{tabular}{|c|c|c|c|c|}
\hline$\vee$ & 0 & $a$ & $b$ & 1 \\
\hline 0 & $\{0\}$ & $\{a\}$ & $\{b\}$ & $\{1\}$ \\
\hline$a$ & $\{a\}$ & $\{0, a\}$ & $\{1\}$ & $\{b, 1\}$ \\
\hline$b$ & $\{b\}$ & $\{1\}$ & $\{0, b\}$ & $\{a, 1\}$ \\
\hline 1 & $\{1\}$ & $\{b, 1\}$ & $\{a, 1\}$ & $L$ \\
\hline
\end{tabular}

Then $(L, \wedge, V)$ is a hyperlattice. Define the maps $d: L \rightarrow L$ by $d x=\left\{\begin{array}{l}0, x=0, a \\ b, x=b, 1\end{array}\right.$ and $D: L \rightarrow L$ by $D x=x$. Then we can see that $D$ is a generalized derivation on $L$.

Definition 2.3 Let $D$ be a generalized derivation on $L$. If $x \leq y$ implies $D x \leq D y$ for all $x, y \in L, D$ is called an isotone generalized derivation. 
Example 2.4 Let $L$ be a hyperlattice as in Example 2.2. It is easy to check that $D$ is an isotone generalized derivation of $L$.

Definition 2.5 A generalized derivation $D$ is said to be contractive if $D x \leq x$ for all $x \in L$.

Proposition 2.6 Let $D$ be a contractive generalized derivation and $d$ be contractive derivation on $L$. Then the following hold for all $x, y \in L$

a) If $L$ is distributive hyperlattice then $d x \leq D x$,

b) If I is a hyperideal of $L$ then $D I \subseteq I$,

c) $D 0=0$

d) $D x \in D x \vee(x \wedge d 1)$

e) $D 1 \in D 1 \vee D 1$

Proof. a) For all $x \in L$, we have

$D x \wedge d x=D(x \wedge x) \wedge d x \in((D x \wedge x) \vee(x \wedge d x)) \wedge d x=(D x \vee d x) \wedge d x$.

Since $L$ is distributive hyperlattice, we obtain

$D x \wedge d x \in(D x \wedge d x) \vee(d x \wedge d x)$. By using Definition $1.1 \quad$ (1) we have $D x \wedge d x \in(D x \wedge d x) \vee d x$. Also from Definition 1.1 (5) we get $(D x \wedge d x) \wedge d x=d x$. Then it is a routine matter to show that $D x \wedge d x=d x$. Consequently we have $d x \leq D x$.

b) Let $y \in D I$. Then there exist an $x \in I$ such that $y=D x \leq x$. Since $I$ is a hyperideal of $L$, we have $y \in I$.

c) It is a routine matter to show that $D 0=D(0 \wedge 0) \in(D 0 \wedge 0) \vee(0 \wedge d 0)$. By using [18] we have $D 0 \in 0$. Hence the result.

d) $D x=D(x \wedge 1) \in(D x \wedge 1) \vee(x \wedge d 1)=D x \vee(x \wedge d 1)$

e) It is clear from Definition 2.1.

Proposition 2.7 Let $D$ be a contractive generalized derivation, then we have

a) If $d 1 \leq x$, then $d 1 \leq D x$,

b) If $x \leq d 1$, then $D x=x$.

Proof. a) Let $x \in L$ such that $d 1 \leq x$, by using Proposition $2.6 d)$ we have $D x \in D x \vee(x \wedge d 1)$ hence $D x \in D x \vee d 1$. Therefore we obtain $d 1 \leq D x$.

b) Let $x \in L$ such that $x \leq d 1$, by using Proposition 2.6 d) we have $D x \in D x \vee(x \wedge d 1)=D x \vee x$, then $x \leq D x$. On the other hand $D$ is a contractive generalized derivation, therefore $D x=x$.

Theorem 2.8 Let $D$ be a contractive generalized derivation on $L$. Then the following conditions are equivalent:

1) $D x=x$ for all $x \in L$,

2) $D(x \vee y)=(x \vee D y) \wedge(D x \vee y)$. 
Proof. $\quad(1) \Rightarrow(2)$ Since $D(x \vee y)=x \vee y$ and $(x \vee D y) \wedge(D x \vee y)=x \vee y$, we get $D(x \vee y)=(x \vee D y) \wedge(D x \vee y)$.

(2) $\Rightarrow(1)$ By putting $x=y$ in (2) we have $D x=x$ for all $x \in L$ since $D$ is contractive generalized derivation.

Theorem 2.9 Let $D$ be a generalized derivation on $L$, then the following conditions are hold.

1) $D$ is an isotone generalized derivation.

2) $D x \vee D y \leq D(x \vee y)$.

Proof. 1) If $x \leq y$, then we get $y \in x \vee y$. Therefore $D y \in D(x \vee y)$. By using Definition 2.1 we have $D y \in D(x \vee y) \subseteq D x \vee D y$. Hence we conclude $D x \leq D y$.

2) Since $D$ is isotone generalized derivation, we have $D x \leq D(x \vee y)$ and $D y \leq D(x \vee y)$. Hence we conclude $D x \vee D y \leq D(x \vee y)$.

\section{Acknowledgements}

The work was supported by grants from CUBAB (F-521).

\section{REFERENCES}

[1] F. Marty, Sur une generalization de la notion de groupe, 8th Congress Math. Scandinaves, Stockholm (1934) 45-49.

[2] R. Ameri, M. Norouzi, New fundamental relation of hyperrings, European J. Combin. 34 (5) (2013) 884-891.

[3] P. Corsini, V. Leoreanu, Applications of Hyperstructure Theory, Kluwer Academic Publishers, Dordrecht (2003).

[4] J. Jantosciak, Transposition hypergroups: noncommutative join spaces. J. Algebra 187(1) (1997) 97119.

[5] J. Zhan, B. Davvaz, P. K. Shun, Probability n-arybypergroups, Information Science 180 (2010) $1159-1166$.

[6] R. Rosaria. Hyperaffine planes over hyperrings, Discrete Math. 155(1-3)(1996) 215-223.

[7] J. Zhan, C. Irina, $\Gamma$-hypermodules:isomorphism theorems and regularrelations, U.P.B. Sci Bull, Ser. A 73 (2011) 71-78.

[8] M. Konstantinidou, J. Mittas. An introduction to the theory of hyperlattices. Math. Balkanica,1977, 7: 187-193.

[9] Mittas, M. Konstantinidou, Sur unenouvellegeneration de la notion de treillis,Lessupertreilliset certaines de leursproprietiesgenerales,AnnSciUnivBlaise Pascal Ser Math, 25 (1989) 61-83.

[10] Xiaozhi Guo, Xiaolong Xin, Hyperlattices, Pure and Applied Mathematics (Xi'an) 20 (2004) 4043.

[11] A. Rahnami-Barghi. The prime ideal theorem for distributive hyperlattices. Ital. J. Pure Appl. Math 10 (2001) 75-78.

[12] S. Rasouli, B. Davvaz, Construction and spectral topology on hyperlattices. Mediterr. J. Math.,7(2) (2010) 249-262.

[13] S. Rasouli, B. Davvaz, Lattices derived from hyperlattices, Commun. Algebra 38 (8) (2010) 27202737.

[14] H. E. Bell, G. Mason,Derivation in Near-Rings, North-Holland Math. Stud., North-Holland, Amsterdam 137 (1987).

[15] E. C. Posner, Derivations in prime rings, Proc. Amer. Math. Soc. 8(1957) 1093-1100. 
[16] M. Bresar, On the distance of composition of the two derivations to the generalized derivations, Glasgow math. J., 33(1), (1991), 89-93.

[17] N. O. Alshehri, Generalized derivations of lattices, Int. J. Contemp. Math. Sciences, 5 (13) (2010) 629-640.

[18] J. Wang, Y. Jun, Xiaolong Xin, Y. Zou, On derivations of bounded hyperlattices, Journal of Mathematical Research and Applications 36(2) (2016) 151-161. 